\title{
Generic features of modulational instability in nonlocal Kerr media
}

\author{
Wyller, John; Krolikowski, Wieslaw; Bang, Ole; Juul Rasmussen, Jens
}

Published in:

Physical Review E. Statistical, Nonlinear, and Soft Matter Physics

Link to article, DOI:

10.1103/PhysRevE.66.066615

Publication date:

2002

Document Version

Publisher's PDF, also known as Version of record

Link back to DTU Orbit

Citation $(A P A)$ :

Wyller, J., Krolikowski, W., Bang, O., \& Juul Rasmussen, J. (2002). Generic features of modulational instability in nonlocal Kerr media. Physical Review E. Statistical, Nonlinear, and Soft Matter Physics, 66(6), 066615 (13 pages). https://doi.org/10.1103/PhysRevE.66.066615

\section{General rights}

Copyright and moral rights for the publications made accessible in the public portal are retained by the authors and/or other copyright owners and it is a condition of accessing publications that users recognise and abide by the legal requirements associated with these rights.

- Users may download and print one copy of any publication from the public portal for the purpose of private study or research.

- You may not further distribute the material or use it for any profit-making activity or commercial gain

- You may freely distribute the URL identifying the publication in the public portal 


\title{
Generic features of modulational instability in nonlocal Kerr media
}

\author{
John Wyller \\ Department of Mathematical Sciences, Agricultural University of Norway, P. O. Box 5065, N-1432 Ås, Norway
}

Wieslaw Krolikowski

Australian Photonics Cooperative Research Centre, Laser Physics Centre, Research School of Physical Sciences and Engineering, Australian National University, Canberra ACT 0200, Australia

Ole Bang

Department of Informatics and Mathematical Modelling, Technical University of Denmark, DK-2800 Kongens Lyngby, Denmark

Jens Juul Rasmussen

Risф National Laboratory, Optics and Fluid Dynamics Department, OFD_128, P. O. Box 49, DK-4000 Roskilde, Denmark

(Received 29 August 2002; published 31 December 2002)

\begin{abstract}
The modulational instability (MI) of plane waves in nonlocal Kerr media is studied for a general response function. Several generic properties are proven mathematically, with emphasis on how new gain bands are formed through a bifurcation process when the degree of nonlocality, $\sigma$, passes certain bifurcation values and how the bandwidth and maximum of each individual gain band depends on $\sigma$. The generic properties of the MI gain spectrum, including the bifurcation phenomena, are then demonstrated for the exponential and rectangular response functions. For a focusing nonlinearity the nonlocality tends to suppress MI, but can never remove it completely, irrespectively of the shape of the response function. For a defocusing nonlinearity the stability properties depend sensitively on the profile of the response function. For response functions with a positivedefinite spectrum, such as Gaussians and exponentials, plane waves are always stable, whereas response functions with spectra that are not positive definite (such as the rectangular) will lead to MI if $\sigma$ exceeds a certain threshold. For the square response function, in both the focusing and defocusing case, we show analytically and numerically how new gain bands that form at higher wave numbers when $\sigma$ increases will eventually dominate the existing gain bands at lower wave numbers and abruptly change the length scale of the periodic pattern that may be observed in experiments.
\end{abstract}

DOI: 10.1103/PhysRevE.66.066615

PACS number(s): 42.65. $-\mathrm{k}$

\section{INTRODUCTION}

The phenomena of modulational instability (MI) of plane waves has been identified and studied in various physical systems, such as fluids [1], plasma [2], nonlinear optics [3,4], discrete nonlinear systems (such as molecular chains [5], Fermi-resonant interfaces, and waveguide arrays [6]), dispersive nonlinear directional couplers with the change of refractive index following a exponential relaxation law [7], etc. It has been shown that MI is strongly affected by various mechanisms present in nonlinear systems, such as higherorder dispersive terms in the case of optical pulses [8], saturation of the nonlinearity [9], and coherence properties of optical beams [10].

In this work we study the MI of plane waves propagating in a nonlinear Kerr-type medium with a nonlinearity $N\left(|\psi|^{2}\right)$ (the refractive index change, in nonlinear optics) that is a nonlocal function of the incident wave field $\psi(x, z)$. We consider a phenomenological model

$$
i \partial_{z} \psi+\frac{1}{2} \partial_{x}^{2} \psi+s N\left(|\psi|^{2}\right) \psi=0
$$

where the nonlinear response $N\left(|\psi|^{2}\right)=R^{*}|\psi|^{2}$ has the generic form of a spatial convolution between the wave intensity $|\psi|^{2}$ and a response function $R(x)$, where $R^{*}|\psi|^{2}$ $=\int_{-\infty}^{\infty} R\left(x^{\prime}-x\right)\left|\psi\left(x^{\prime}, z\right)\right|^{2} d x^{\prime}$, Here $x$ is the transverse spatial coordinate and $s=1(s=-1)$ corresponds to a focusing (defocusing) nonlinearity. The evolution coordinate $z$ can be the time coordinates, as for Bose-Einstein condensates, or the propagation coordinate, as for optical beams. We consider only symmetric spatial response functions that are positive definite and (without loss of generality) obey the normalization condition

$$
\int_{-\infty}^{\infty} R(x) d x=1 .
$$

Thus we exclude asymmetric effects, such as those generated by asymmetric temporal response functions (with $x$ being time), as in the case of the Raman effect on optical pulses [11].

In nonlinear optics, Eq. (1) represents a general phenomenological model for media in which the nonlinear refractive index change (or polarization) induced by an optical beam is determined by some kind of a transport process. It may include, e.g., heat conduction in materials with a thermal nonlinearity [12-15] or diffusion of molecules or atoms accompanying nonlinear light propagation in atomic vapors [16]. Nonlocality also accompanies the propagation of waves in plasma [15,17-21], and a nonlocal response in form (1) appears naturally as a result of many-body interaction pro- 
cesses in the description of Bose-Einstein condensates [22]. The orientational nonlinearity of liquid crystals is also nonlocal and may be described by Eq. (1) [23,24]. It is worth to mention that an important aspect of the nonlocality in all these systems is that it completely prevents collapse of finitesize beams [25].

The width of the response function $R(x)$ relative to the width of the intensity profile $|\psi(x, z)|^{2}$ determines the degree of nonlocality. In the limit of a singular response we get the well-known nonlinear Schrödinger (NLS) equation that appears in all areas of physics. Here the focusing case $(s=1)$ produces MI of the finite bandwidth type, while the defocusing case $(s=-1)$ predicts modulational stability [3]. When the width of the response function is finite but small compared to that of the intensity distribution, model (1) is approximated by the weakly nonlocal NLS equation [26-30]

$$
i \partial_{z} \psi+\frac{1}{2} \partial_{x}^{2} \psi+s\left[|\psi|^{2}+\gamma \partial_{x}^{2}|\psi|^{2}\right] \psi=0
$$

Here $\gamma \ll 1$ is defined as the second virial of $R(x)$,

$$
\gamma \equiv \frac{1}{2} \int_{-\infty}^{\infty} x^{2} R(x) d x
$$

In contrast to the local NLS limit $(\gamma=0)$, the MI now depends not only on the sign of $s$ but also on the intensity of the plane waves [18]. Finally, in the case of strong nonlocality it has been shown that Eq. (1) simplifies to a linear model, and hence there is no MI in this limit [31].

MI has thus been studied in different limits. The general case (1) has recently been investigated with respect to MI and compared with the weakly nonlocal limit [26]. Here we present an analytical study of the full nonlocal case with arbitrary profile $R(x)$ whose spectrum obeys a sufficient degree of smoothness, with particular emphasis on generic features of the MI. The present paper complements and extends the results obtained in Ref. [26].

\section{MI IN THE NONLOCAL NLS EQUATION}

Model (1) has plane wave solutions of the form

$$
\psi(x, z)=\sqrt{\rho_{0}} \exp \left(i k_{0} x-i \omega_{0} z\right), \quad \rho_{0}>0,
$$

where $\rho_{0}, k_{0}$, and $\omega_{0}$ are linked through the nonlinear dispersion relation

$$
\omega_{0}=\frac{1}{2} k_{0}^{2}-s \rho_{0},
$$

Following Ref. [26], we perturb the plane wave solutions as follows:

$$
\begin{gathered}
\psi(x, z)=\left[\sqrt{\rho_{0}}+u(\xi, \tau)+i v(\xi, \tau)\right] \exp \left(i \theta_{0}\right), \\
\xi=x-k_{0} z, \quad \tau=z, \quad \theta_{0}=k_{0} x-\omega_{0} z,
\end{gathered}
$$

TABLE I. Existence criteria for MI in nonlocal media.

\begin{tabular}{lcc}
\hline \hline & $s=+1$ & $s=-1$ \\
\hline$\hat{R}(k)>0$ & MI & Stability \\
$\hat{R}(k)$ not sign definite & MI & depends on $\hat{R}(k)$ \\
\hline \hline
\end{tabular}

where $u$ and $v$ are real functions. Inserting this expression into the nonlocal NLS equation (1) and linearizing around the solution (5)-(6) gives the equations

$$
\begin{gathered}
\partial_{\tau} u+\frac{1}{2} \partial_{\xi}^{2} v=0, \\
\partial_{\tau} v-\frac{1}{2} \partial_{\xi}^{2} u-2 s \rho_{0}\left(R^{*} u\right)=0,
\end{gathered}
$$

where $R^{*} u=\int_{-\infty}^{\infty} R\left(\xi^{\prime}-\xi\right) u\left(\xi^{\prime}, \tau\right) d \xi^{\prime}$ is again the spatial convolution integral. Introducing the spatial Fourier transform of a function as $\widehat{f}(k)=\int_{-\infty}^{\infty} f(\xi) \exp (i k \xi) d \xi$, and exploiting the convolution theorem for Fourier transforms, the linearized system is converted to a set of ordinary differential equations in $k$ space,

$$
\partial_{\tau} X=\underline{A X}
$$

where the vector $X$ and matrix $A$ are defined as

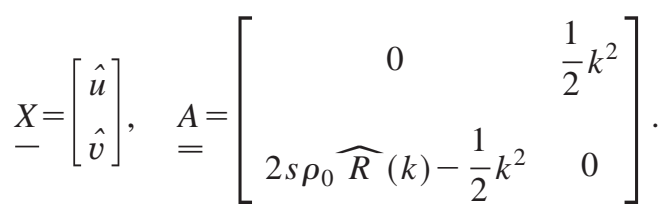

The eigenvalues $\lambda$ of the matrix $A$ are given by

$$
\lambda^{2}=k^{2} \rho_{0}\left[s \widehat{R}(k)-\frac{1}{4 \rho_{0}} k^{2}\right] .
$$

The general expression (12) was also derived in Ref. [26]. Here we have briefly reviewed the derivation for the sake of completenes and because it constitutes the basis of our study of MI. From Eq. (12) one can deduct the general existence properties of MI in nonlocal media listed in Table I [26].

\section{GENERIC PROPERTIES OF THE MI GAIN SPECTRUM}

The local case with $\sigma=0$ (the NLS equation) is well known. There is always MI in focusing media $(s=1)$ and always stability in defocusing media $(s=-1)$. Here we therefore focus on the interesting case with a finite degree of nonlocality $(\sigma \neq 0)$. The spectrum $\hat{R}(k)$ has the following generic properties:

(1) Since $R(x)$ is real valued and symmetric, then so is $\hat{R}(k)$, i.e., $\hat{R}(k)=\hat{R}(-k)=\hat{R}^{*}(k)$.

(2) Normalization (2) implies that $\hat{R}(0)=1$, which means that $R(x)$ tacitly is assumed to be absolutely integrable, and 
hence $\hat{R}(k)$ is continuous [32].

(3) Symmetry condition 1 . imposes $\hat{R}^{\prime}(0)=0$, i.e., the spectrum $\hat{R}(k)$ has a critical point at $k=0$, provided $\hat{R}(k)$ is a differentiable function of $k$. Here and in the following, prime denotes differentiation with respect to the argument.

(4) Normalization (2) for $R(x)$ means that $R(x)$ is absolute integrable, and hence by Riemann-Lebesque lemma we have $\lim _{|k| \rightarrow \infty} \hat{R}(k)=0$ [32].

We further assume the following properties:

(5) The functions $x R(x)$ and $x^{2} R(x)$ are also absolutely integrable, which means that $\hat{R}^{\prime}(k)$ and $\hat{R}^{\prime \prime}(k)$ are continuous for all $k$ [32].

(6) The response function is characterized by a typical width or scaling length $\sigma$ (the degree of nonlocality) and assume the generic form $R(x)=\sigma^{-1} \Phi(x / \sigma)$, where the scaling function $\Phi$ is nondimensional, i.e., independent of $\sigma$.

The spectrum $\hat{R}(k)$ can be expressed in terms of the Fourier transform $\hat{\Phi}$ of the scaling function $\Phi$ as

$$
\hat{R}(k)=\hat{\Phi}(\sigma k) .
$$

The properties (1)-(6) of the spectrum $\hat{R}$ carry over to $\hat{\Phi}$.

Note that due to the normalization [property (2)] and the Riemann-Lebesque lemma [property (4)], the scaling function has the property that

$$
\lim _{\sigma \rightarrow \infty} \hat{\Phi}(\sigma k)= \begin{cases}1, & k=0 \\ 0, & k \neq 0 .\end{cases}
$$

Thus eigenvalue equation (12) approaches uniformly the form $\lambda^{2}=-k^{4} / 4$ for $\sigma \rightarrow \infty$, which is recognized as the eigenvalue equation for MI in the linear potential free Schrödinger equation

$$
i \partial_{z} \psi+\frac{1}{2} \partial_{x}^{2} \psi=0
$$

Hence the well-known result of modulational stability is reproduced in the limit of strong nonlocality.

For $0<\sigma<\infty$ we proceed as follows: Using the nondimensional scaling function $\hat{\Phi}$, the degree of nonlocality can be explicitly put into eigenvalue equation (12), even though the specific response function is not known. We thus rewrite Eq. (12) in the form

$$
\left(\frac{\lambda}{2 \rho_{0}}\right)^{2}=\bar{k}^{2} \phi(\bar{k}, \bar{s})
$$

where the scaled wave number $\bar{k}$ and the scaled nonlinearity $\bar{s}$ are defined as

$$
\bar{k}=\sigma k, \quad \bar{s}=4 \rho_{0} \sigma^{2} s .
$$

The MI gain band structure is then determined by the structural function $\phi(\bar{k}, \bar{s})$, given by

$$
\phi(\bar{k}, \bar{s}) \equiv \frac{\bar{s} \hat{\Phi}(\bar{k})-\bar{k}^{2}}{\bar{s}^{2}} .
$$

This is the most convenient form for the mathematical treatment. Due to the symmetry property (1), we consider only positive wave numbers in the following, i.e., $\bar{k} \geqslant 0$.

The parameter $\bar{s}$ contains both the nonlinearity and the degree of nonlocality, and plays the role of a control parameter. The crucial point in the MI analysis is the properties of the function $\phi$ in the $(\bar{k}-\bar{s})$ space. Using properties (1)-(6) we can characterize the set $\Omega$ of (unstable wave numbers) $\bar{k}$ fulfilling the inequality $\phi(\bar{k}, \bar{s}) \geqslant 0$ for a given value of $\bar{s}$ as follows:

(I) Modulational stability. If $\phi(\bar{k}, \bar{s}) \leqslant 0$ for all $\bar{k}$ then $\Omega$ is empty. In this case there can be no MI.

(II) Finite bandwidth MI.

(a) If $\phi(\bar{k}, \bar{s}) \geqslant 0$ for any $\bar{k}$ then MI occurs. For such $\bar{k}$ values we define the normalized MI growth rate $g$,

$$
g=\left|\frac{\lambda}{2 \rho_{0}}\right|=|\vec{k}| \sqrt{\phi(\bar{k}, \bar{s})} .
$$

(b) Any MI gain spectrum will be of finite bandwidth because $\hat{\Phi}$ is localized [property (4)] and thus $\phi \rightarrow-\bar{k}^{2} / \vec{s}^{2}$ for $|\bar{k}| \rightarrow \infty$.

(c) The number of MI gain bands is generically finite. This follows if one can show that the transversality condition $\partial_{\bar{k}} \phi \neq 0$ is satisfied at all the zeros of $\phi$ for a given $\bar{s}$. This result is proved in the Appendix (Theorem I).

(d) The breakdown of the transversality condition for certain values of the control parameter $\bar{s}$, i.e., $\partial_{\bar{k}} \phi=\phi=0$, describes bifurcation phenomena like excitation, vanishing, coalescence, and separation of MI bands.

This list represents the overall picture of the MI for the nonlocal NLS equation (1). In the following we detail different aspects of this picture. We first formulate the theory of excitation, vanishing, coalescence, and separation of MI bands as bifurcation phenomena. Then we study the general properties of the focusing case $(\bar{s}>0)$ and the defocusing case $(\bar{s}$ $<0$ ), separately. Finally, the discussion is illuminated with examples.

\section{BIFURCATION ANALYSIS: EXCITATION, VANISHING, MERGING, AND SEPARATION OF MI GAIN BANDS}

Let $\left(\bar{k}_{b}, \bar{s}_{b}\right) \neq(0,0)$ denote points where the transversality condition breaks down, i.e., where the structural function $\phi$ and its derivative $\partial_{\bar{k}} \phi$ both are zero,

$$
\phi\left(\bar{k}_{b}, \bar{s}_{b}\right)=\partial_{\bar{k}} \phi\left(\bar{k}_{b}, \bar{s}_{b}\right)=0 .
$$

The number of zeros of $\phi$, and thus the number of gain bands, will change as $\bar{s}$ passes the bifurcation value $\bar{s}_{b}$. The second derivative of the structural function evaluated at the bifurcation point determines what type of phenomenon takes 
place. If the second derivative is positive, then two MI gain bands merge, or one band separates into two,

$$
\partial_{\bar{k}}^{2} \phi\left(\bar{k}_{b}, \bar{s}_{b}\right)>0 \Rightarrow \text { merging or separation, }
$$

while a negative second derivative represents excitation or vanishing of an MI gain band,

$$
\partial_{\bar{k}}^{2} \phi\left(\bar{k}_{b}, \bar{s}_{b}\right)<0 \Rightarrow \text { excitation or vanishing. }
$$

In fact, in a similar way as in the proof of Theorem I, it can be shown that the bifurcation points $\left(\bar{k}_{b}, \bar{s}_{b}\right)$ are distinct and isolated if the condition

$$
\partial_{\bar{k}}^{2} \phi\left(\bar{k}_{b}, \bar{s}_{b}\right) \neq 0
$$

is satisfied. This is a key result in our theory. The nongeneric case $\partial_{\bar{k}}^{2} \phi\left(\bar{k}_{b}, \bar{s}_{b}\right)=0$ will not be considered here. Finally, an overall requirement for a bifurcation to actually take place is that the velocity condition

$$
\partial_{s}^{-} \phi\left(\bar{k}_{b}, \bar{s}_{b}\right) \neq 0
$$

is satisfied at the bifurcation point. This assures that $\phi\left(\bar{k}_{b}, \bar{s}\right)$ does not remain on the zero axis, but crosses it when the control parameter $\bar{s}$ passes through the bifurcation value $\bar{s}_{b}$. Conditions (18), (21), and (22) are referred to as the generic conditions for bifurcations.

Using the definition (16) of the structural function $\phi$, we can find more specific results. Thus Eq. (18) becomes

$$
\begin{gathered}
\bar{k}_{b} \hat{\Phi}^{\prime}\left(\bar{k}_{b}\right)=2 \hat{\Phi}\left(\bar{k}_{b}\right), \quad \bar{k}_{b}>0, \\
\bar{s}_{b}=\bar{k}_{b}^{2} / \hat{\Phi}\left(\bar{k}_{b}\right) .
\end{gathered}
$$

By assumption $\bar{s}_{b} \neq 0$, and thus Eq. (24) implies $\bar{k}_{b} \neq 0$. To determine the bifurcation values $\bar{k}_{b}$ and $\bar{s}_{b}$ of the scaled wave number and the control parameter, one first solves Eq. (23) for $\bar{k}_{b}$ under the condition $s \hat{\Phi}\left(\bar{k}_{b}\right)>0$. Then $\bar{s}_{b}$ is given by Eq. (24). The second derivative is given by

$$
\partial_{\bar{k}}^{2} \phi\left(\bar{k}_{b}, \bar{s}_{b}\right)=\frac{\bar{s}_{b} \hat{\Phi}^{\prime \prime}\left(\bar{k}_{b}\right)-2}{\bar{s}_{b}^{2}},
$$

and thus the generic condition (21) may be written as

$$
\bar{s}_{b} \hat{\Phi}^{\prime \prime}\left(\bar{k}_{b}\right)=\bar{k}_{b}^{2} \frac{\hat{\Phi}^{\prime \prime}\left(\bar{k}_{b}\right)}{\hat{\Phi}\left(\bar{k}_{b}\right)} \neq 2 .
$$

Importantly, we find that at the bifurcation point,

$$
\partial_{s}^{-} \phi\left(\bar{k}_{b}, \bar{s}_{b}\right)=\bar{k}_{b}^{2} / \bar{s}_{b}^{3} \neq 0
$$

The velocity condition (22) is therefore always satisfied in our general nonlocal system (1). In Table II we have summarized the features of the bifurcation process.
TABLE II. Generic conditions for excitation, vanishing, merging, and separation of MI gain bands in the nonlocal NLS equation (1).

\begin{tabular}{l|c}
\hline \hline Generic condition & Characteristic feature \\
\hline $\bar{k}_{b}^{2} \frac{\hat{\Phi}^{\prime \prime}\left(\bar{k}_{b}\right)}{\hat{\Phi}\left(\bar{k}_{b}\right)}>2$ & Separation or merging \\
\hline $\bar{k}_{b}^{2} \frac{\hat{\Phi}^{\prime \prime}\left(\bar{k}_{b}\right)}{\hat{\Phi}\left(\bar{k}_{b}\right)}<2$ & Excitation or vanishing \\
\hline \hline
\end{tabular}

\section{MI GAIN SPECTRUM IN FOCUSING MEDIA}

To obtain specific results (bandwidth, maximum, etc.) about the individual gain bands constituting the normalized MI gain spectrum (17), we now consider the focusing case $\bar{s}>0$. We apply symmetry property (1) $[\hat{\Phi}(\bar{k})=\hat{\Phi}(-\bar{k})]$ and consider only positive wave numbers $\bar{k} \geqslant 0$. In this case the following features are apparent:

(i) Existence of a fundamental gain band. There exists a closed bounded interval $\left[0, \bar{k}_{1}\right]$ in which $\phi(\bar{k}, \bar{s})>0$ for 0 $\leqslant \bar{k}<\bar{k}_{1}$ and $\phi\left(\bar{k}_{1}, \bar{s}\right)=0$. This follows from the properties (2)-(4) and the intermediate value theorem for continuous functions. Hence there will always be MI, independent of the shape of the spectrum $\hat{\Phi}$. The band $\left[0, \bar{k}_{1}\right]$ originates from the underlying local NLS equation, and is thus denoted as the fundamental gain band.

(ii) Possible existence of higher-order gain bands. MI gain bands may also exist for wave numbers $\bar{k}>\bar{k}_{1}$. This property depends sensitively on the shape of the spectrum and on the control parameter $\bar{s}$.

(iii) Number of gain bands. If the transversality condition $\partial_{\bar{k}} \phi \neq 0$ is satisfied at all the $N$ zeros of $\phi$ for a given $\bar{s}$ $>0$, then the number of gain bands, $m$, is given by $N=2 m$ $-1(m=1,2, \ldots)$. This follows from Theorem I in the Appendix, and the fact that $\phi(0, \bar{s})=1 / \bar{s}>0$ and $\phi \rightarrow-\bar{k}^{2} / \bar{s}^{2}$ for $\bar{k} \rightarrow \infty$.

\section{A. The fundamental gain band}

The fundamental gain band exists for all values of the degree of nonlocality, $\sigma$ (i.e., of the control parameter $\bar{s}$ ), also in the local limit described by the focusing NLS equation. This gain band has the following features:

(f1) Gain profile. If the transversality condition $\partial_{\bar{k}} \phi\left(\bar{k}_{1}, \bar{s}\right)<0$ [i.e., $\hat{\Phi}^{\prime}\left(\bar{k}_{1}\right)<2 \bar{k}_{1} / \bar{s}$ ] is satisfied, then there is at least one critical point $\bar{k}_{c} \in\left[0, \bar{k}_{1}\right]$ at which $\partial_{\bar{k}} g\left(\bar{k}_{c}, \bar{s}\right)$ $=0$. This follows from the fact that $\partial_{\bar{k}} g(0, \bar{s})=1 / \sqrt{\bar{s}}$, $\partial_{\bar{k}} g(\bar{k}, \bar{s}) \rightarrow-\infty$ as $\bar{k} \rightarrow \bar{k}_{1}^{-}$and the intermediate value theorem for continuous functions. [It is possible to extend this argument to cases where the spectrum $\hat{\Phi}$ does not possess a well-defined tangent at $\bar{k}=0$. If we require that $\lim _{\bar{k} \rightarrow 0^{+}} \hat{\Phi}^{\prime}(\bar{k})$ exists (but is different from zero), then we also have existence of at least one critical point $\bar{k}_{c} \in\left[0, \bar{k}_{1}\right]$. This occurs, for example, for the spectrum of the Lorentzian 
response function.] At least one of these critical points $\bar{k}_{c}$ gives a maximum growth rate $g_{\max }(\bar{s})$. The curvature of the gain spectrum is given by

$$
\partial_{\bar{k}}^{2} g\left(\bar{k}_{c}, \bar{s}\right)=\frac{F\left(\bar{k}_{c}, \bar{s}\right)}{2 \sqrt{\phi\left(\bar{k}_{c} ; \bar{s}\right)}},
$$

where we have defined the functions

$$
\begin{gathered}
F(\bar{k}, \bar{s})=\bar{s} D(\bar{k})-8 \bar{k}, \\
D(\bar{k})=3 \hat{\Phi}^{\prime}(\bar{k})+\bar{k} \hat{\Phi}^{\prime \prime}(\bar{k}) .
\end{gathered}
$$

Thus the gain curve has a local minimum at $\bar{k}_{c}$ if $F\left(\bar{k}_{c}, \bar{s}\right)$ $>0$ and a local maximum if $F\left(\bar{k}_{c}, \bar{s}\right)<0$. A special case occurs if the curvature is negative for all $\bar{k}_{c}$, i.e., if $F\left(\bar{k}_{c}, \bar{s}\right)<0$ is always satisfied. Then the maximum is unique, i.e., only one critical wave number $\bar{k}_{c}$ exists. Restoring to original variables, the normalized gain is given by

$$
g=\frac{k}{4 \rho_{0}} \sqrt{4 \rho_{0} \hat{\Phi}(\bar{k})-k^{2}} .
$$

For fixed amplitude $\rho_{0}$ the velocity therefore becomes

$$
\frac{\partial g}{\partial \sigma}=\left(\frac{k^{3}}{8 \rho_{0} g}\right) \hat{\Phi}^{\prime}(\bar{k}),
$$

and thus the variation of the gain with the degree of nonlocality $\sigma$ depends on the sign of the function $\hat{\Phi}^{\prime}\left(\bar{k}_{1}\right)$, i.e., $\operatorname{sgn}\{\partial g / \partial \sigma\}=\operatorname{sgn}\left\{\hat{\Phi}^{\prime}\left(\bar{k}_{1}\right)\right\}$.

(f2) Bandwidth. The condition $\phi\left[\bar{k}_{1}(\bar{s}), \bar{s}\right]=0$ defines the bandwidth $\bar{k}_{1}(\bar{s})$ as a function of the control parameter, from which we find the velocity

$$
\frac{\partial \bar{k}_{1}}{\partial \bar{s}}=-\frac{\bar{k}_{1}^{2}}{\bar{s}\left[\bar{s} \hat{\Phi}^{\prime}\left(\bar{k}_{1}\right)-2 \bar{k}_{1}\right]} .
$$

Since the transversality condition $\hat{\Phi}^{\prime}\left(\bar{k}_{1}\right)<2 \bar{k}_{1} / \bar{s}$ is satisfied, there is no problem with division by zero, and this velocity is always positive. For fixed amplitude $\rho_{o}$, this expression becomes

$$
\frac{\partial k_{1}}{\partial \sigma}=-\frac{2 \rho_{0} k_{1} \hat{\Phi}^{\prime}\left(\bar{k}_{1}\right)}{2 \rho_{0} \sigma \hat{\Phi}^{\prime}\left(\bar{k}_{1}\right)-k_{1}}
$$

in real variables. The transversality condition implies that $2 \rho_{0} \sigma \hat{\Phi}^{\prime}\left(\bar{k}_{1}\right)<k_{1}$, and thus the variation of the bandwidth with the degree of nonlocality $(\sigma)$ depends on the sign of $\hat{\Phi}^{\prime}\left(\bar{k}_{1}\right)$, i.e., $\operatorname{sgn}\left\{d k_{1} / d \sigma\right\}=\operatorname{sgn}\left\{\hat{\Phi}^{\prime}\left(\bar{k}_{1}\right)\right\}$. For a fixed degree of nonlocality $(\sigma)$, we obtain

$$
\frac{\partial k_{1}}{\partial \rho_{0}}=-\frac{k_{1}^{2} /\left(2 \rho_{0}\right)}{2 \rho_{o} \sigma \hat{\Phi}^{\prime}\left(\bar{k}_{1}\right)-k_{1}}>0 .
$$

Thus the bandwidth $k_{1}$ always increases with increasing amplitude of the plane wave, $\rho_{0}$ (i.e., the more nonlinear the system is), no matter what the degree of nonlocality is.

(f3) Local minima and maxima. The criticality condition $\partial_{\bar{k}} g\left(\bar{k}_{c}, \bar{s}\right)=0$ is equivalent to the equation

$$
H\left[\bar{k}_{c}, \bar{s}\right]=0,
$$

which determines the critical wave number $\bar{k}_{c}(\bar{s})$ as a function of the control parameter $\bar{s}$. The function

$$
H(\bar{k}, \bar{s}) \equiv \bar{s}\left[2 \hat{\Phi}(\bar{k})+\bar{k} \hat{\Phi}^{\prime}(\bar{k})\right]-4 \bar{k}^{2}
$$

satisfies the relation $d H / d \bar{s}=F d \bar{k} / d \bar{s}$. Thus, for $F\left(\bar{k}_{c}, \bar{s}\right)$ $\neq 0$ (nonzero gain curvature), we obtain the velocity

$$
\frac{d \bar{k}_{c}}{d \bar{s}}=-\frac{4 \bar{k}_{c}^{2}}{\bar{s} F\left(\bar{k}_{c}, \bar{s}\right)}
$$

For fixed amplitude $\rho_{0}$, this expression becomes

$$
\frac{\partial k_{c}}{\partial \sigma}=-\frac{k_{c} \rho_{0} D\left(\bar{k}_{c}\right)}{\sigma \rho_{0} D\left(\bar{k}_{c}\right)-2 k_{c}} .
$$

From Eq. (38), we see that if the degree of nonlocality $\sigma$ increases, then a local minimum $\left[\sigma \rho_{0} D\left(\bar{k}_{c}\right)>2 k_{c}\right]$ will always decrease, whereas the properties of a local maximum $\left[\sigma \rho_{0} D\left(\bar{k}_{c}\right)<2 k_{c}\right]$ will depend on the sign of the function $D\left(\bar{k}_{c}\right)$. If $0<D\left(\bar{k}_{c}\right)<8 \bar{k}_{c} / \bar{s}$ then $k_{c}$ will increase, and if $D\left(\bar{k}_{c}\right)<0$ then $k_{c}$ will decrease.

At inflection points, $F\left(\bar{k}_{c}, \bar{s}\right)=0\left[\sigma \rho_{0} D\left(\bar{k}_{c}\right)=2 k_{c}\right]$, and thus Eq. (38) does not apply. An inflection point actually corresponds to a bifurcation, at which two critical points (extrema of the gain curve), i.e., one local minimum and one local maximum, will be formed or vanish. The bifurcation points $\left(\bar{k}_{c}, \bar{s}\right)=\left(\bar{k}_{e}, \bar{s}_{e}\right)$ are solutions of the system of equations $F\left(\bar{k}_{e}, \bar{s}_{e}\right)=H\left(\bar{k}_{e}, \bar{s}_{e}\right)=0$, which gives

$$
G\left(\bar{k}_{e}\right)=\bar{k}_{e}^{2} \hat{\Phi}^{\prime \prime}\left(\bar{k}_{e}\right)+\bar{k}_{e} \hat{\Phi}^{\prime}\left(\bar{k}_{e}\right)-4 \hat{\Phi}\left(\bar{k}_{e}\right)=0
$$

and $\bar{s}_{e}=8 \bar{k}_{e} / D\left(\bar{k}_{e}\right)$. The bifurcation points are isolated and distinct if the transversality condition $d G / d \bar{k}_{e} \neq 0$ is satisfied.

For typical response functions with a positive, monotonically decaying spectrum (such as the Lorentzian spectrum commonly appearing in physical problems), i.e., with $\hat{\Phi}^{\prime}(\bar{k})<0$ for all $\bar{k}$, both the gain $g(k)$ and the bandwidth $k_{1}$ will always be a decreasing function of the degree of nonlocality. If each gain band further has a unique maximum, then bifurcations will not be an issue. Table III summarizes the generic features of the fundamental gain band in nonlocal focusing media $(\bar{s}>0)$. Note that the fundamental gain band always has the classical Lighthill shape of the MI gain spectrum in the local focusing NLS equation. 
TABLE III. Generic features of the fundamental gain band of the MI gain spectrum $g$ in nonlocal focusing media $(\bar{k}=\sigma k \geqslant 0, \bar{s}$ $\left.=4 \rho_{0} \sigma^{2}>0\right)$ with the degree of nonlocality $(\sigma)$.

\begin{tabular}{|c|c|}
\hline Generic condition & Characteristic feature \\
\hline None & $\begin{array}{l}\text { Finite bandwidth } k_{1} \text {, where } \\
\qquad \bar{s} \hat{\Phi}\left(\bar{k}_{1}\right)=\bar{k}_{1}^{2} .\end{array}$ \\
\hline$\hat{\Phi}^{\prime}\left(\bar{k}_{1}\right)<0(>0)$ & $\begin{array}{l}\text { The bandwidth decreases } \\
\text { (increases) with } \sigma \text {. }\end{array}$ \\
\hline$\hat{\Phi}^{\prime}\left(\bar{k}_{1}\right)<2 \bar{k}_{1} / \bar{s}$ & $\begin{array}{c}g \simeq k / \sqrt{4 \rho_{0}} \text { as } k \rightarrow 0 \\
\partial_{k} g \rightarrow-\infty \text { as } k \rightarrow k_{1}^{-} \\
\text {A maximum gain exists. }\end{array}$ \\
\hline $\begin{array}{l}D\left(\bar{k}_{c}\right)>8 \bar{k}_{c} / \bar{s} \\
D(\bar{k})=3 \hat{\Phi}^{\prime}(\bar{k})+\bar{k} \hat{\Phi}^{\prime \prime}(\bar{k})\end{array}$ & $\begin{array}{c}\text { Local minimum at } k_{c}, \partial_{\bar{k}} g\left(\bar{k}_{c}\right)=0, \\
\text { which decreases with } \sigma .\end{array}$ \\
\hline$D\left(\bar{k}_{c}\right)=8 \bar{k}_{c} / \bar{s}_{e}$ & $\begin{array}{l}\text { Inflection point at } k_{c} . \text { When } \bar{s} \\
\text { passes } \bar{s}_{e} \text {, two local extrema (a } \\
\text { minimum and a maximum) will } \\
\text { form or vanish. }\end{array}$ \\
\hline $0<D\left(\bar{k}_{c}\right)<8 \bar{k}_{c} / \bar{s}$ & $\begin{array}{l}\text { Local maximum at } k_{c} \text {, which } \\
\text { increases with } \sigma .\end{array}$ \\
\hline$D\left(\bar{k}_{c}\right)<0$ & $\begin{array}{l}\text { Local maximum at } k_{c} \text {, which } \\
\text { decreases with } \sigma \text {. }\end{array}$ \\
\hline $\begin{array}{l}D(\bar{k})<8 \bar{k} / \bar{s} \\
\text { for all } \bar{k}\end{array}$ & $\begin{array}{l}\text { One unique maximum. No local } \\
\text { minima or inflection points. }\end{array}$ \\
\hline$\hat{\Phi}^{\prime}(\bar{k})<0$ for $0<\bar{k} \leqslant \bar{k}_{1}$ & $\begin{array}{l}\text { The gain and bandwidth } \\
\text { decreases with } \sigma \text {. }\end{array}$ \\
\hline
\end{tabular}

\section{B. Higher-order gain bands}

Here we detail the properties of the higher-order gain bands that do not exist in the local limit $\sigma=0$, but may be formed through a bifurcation process when the system becomes sufficiently nonlocal. Let $\bar{s}_{b}$ denote the bifurcational value of the control parameter and assume that for $\bar{s}>\bar{s}_{b}$ there are wave numbers $\bar{k}_{0}$ and $\bar{k}_{1}\left(\bar{k}_{0}<\bar{k}_{1}\right)$ satisfying the following properties $(i=0,1)$ :

(a) $\phi\left(\bar{k}_{i}, \bar{s}\right)=0 \Leftrightarrow \hat{\Phi}\left(\bar{k}_{i}\right)=\bar{k}_{i}^{2} / \bar{s}$.

(b) $\phi(\bar{k}, \bar{s})>0$ for $\bar{k}_{0}<\bar{k}<\bar{k}_{1} \Leftrightarrow \hat{\Phi}(\bar{k})>\bar{k}^{2} / \bar{s}$ for $\bar{k}_{0}<$ $\bar{k}<\bar{k}_{1}$.

(c) $\partial_{\bar{k}} \phi\left(\bar{k}_{0}, \bar{s}\right)>0 \Leftrightarrow \hat{\Phi}^{\prime}\left(\bar{k}_{0}\right)>2 \bar{k}_{0} / \bar{s}$.

(d) $\partial_{\bar{k}} \phi\left(\bar{k}_{1}, \bar{s}\right)<0 \Leftrightarrow \hat{\Phi}^{\prime}\left(\bar{k}_{1}\right)<2 \bar{k}_{1} / \bar{s}$.

Note that assumptions (c) and (d) imply that we have imposed transversality conditions at $\bar{k}_{0}$ and $\bar{k}_{1}$. The following results are apparent:

(hf1) Gain profile. The gain $g$ is positive and continuous differentiable for $\bar{k}_{0}<\bar{k}<\bar{k}_{1}$. At the boundaries, $g\left(\bar{k}_{0}, \bar{s}\right)$ $=g\left(\bar{k}_{1}, \bar{s}\right)=0, \partial_{\bar{k}} g\left(\bar{k}_{0}, \bar{s}\right) \rightarrow \infty$ for $\bar{k} \rightarrow \bar{k}_{0}^{+}$, and $\partial_{\bar{k}} g\left(\bar{k}_{1}, \bar{s}\right)$ $\rightarrow-\infty$ for $\bar{k} \rightarrow \bar{k}_{1}^{-}$. Hence, by the intermediate value theorem, there is at least one critical wave number $\bar{k}_{c} \in\left[\bar{k}_{0}, \bar{k}_{1}\right]$ at which the gain has a local extremum, $\partial_{\bar{k}} g\left(\bar{k}_{c}, \bar{s}\right)=0$. Equations (27)-(31) and the properties (f1) derived therefrom all apply to the higher-order gain bands also, e.g., local minima, maxima, and inflection points are distinguished by the sign of the function $F\left(\bar{k}_{c}\right)$.

(hf2) Bandwidth. The bandwidth of the higher order gain bands is defined as $B \equiv k_{1}-k_{0}$. The transversality conditions (c) and (d) mean that Eqs. (32)-(34) for the velocities apply to both the boundaries $k_{0}$ and $k_{1}$, i.e., to $\partial k_{i} / \partial \sigma$ and $\partial k_{i} / \partial \rho_{0}$. Thus the left boundary $k_{0}$ will always decrease with the degree of nonlocality $(\sigma)$, since $\hat{\Phi}^{\prime}\left(\bar{k}_{0}\right)$ $>k_{0} /\left(2 \rho_{0} \sigma\right)$. The variation of the right boundary $k_{1}$ with $\sigma$ will depend on the sign of the function $\hat{\Phi}^{\prime}\left(\bar{k}_{1}\right)$, as for the fundamental gain band. However, even if $\hat{\Phi}^{\prime}\left(\bar{k}_{1}\right)<0$ and $k_{1}$ therefore decreases with $\sigma$, the bandwidth $B$ may still increase with $\sigma$ if $k_{0}$ decreases faster than $k_{1}$, i.e., if $\partial k_{0} / \partial \sigma$ $<\partial k_{1} / \partial \sigma$

In terms of the plane-wave amplitude $\rho_{0}$, the right boundary $k_{1}$ will always increase with $\rho_{0}$ as for the fundamental gain band $\left(\partial k_{1} / \partial \rho_{0}>0\right)$, whereas the left boundary $k_{0}$ will always decrease with $\rho_{0}\left(\partial k_{0} / \partial \rho_{0}>0\right)$ due to the conditions (c) and (d). Thus the bandwidth $B$ will increase rapidly the stronger the focusing nonlocal nonlinearity.

(hf3) Local minima and maxima. Equations (35)-(38) and the properties (f3) of local minima and maxima in the fundamental gain band also apply to the higher-order gain bands.

\section{MI GAIN SPECTRUM IN DEFOCUSING MEDIA}

We now consider the defocusing case with $\bar{s}<0$ and apply again the symmetry property (1) $[\hat{\Phi}(\bar{k})=\hat{\phi}(-\bar{k})]$ to consider only positive wave numbers $\bar{k} \geqslant 0$. The following main features are apparent:

(i) Nonexistence of the fundamental gain band. No closed bounded interval $\left[0, k_{1}\right]$ exists, for which $\phi(\bar{k}, \bar{s})>0$ when $0 \leqslant \bar{k}<\bar{k}_{1}$ and $\phi\left(\bar{k}_{1}, \bar{s}\right)=0$. This follows from the fact $\phi(0, \bar{s})<0$ and continuity of the structural function.

(ii) Modulational stability for positive definite spectra. If $\hat{\Phi}(\bar{k})>0$ for all $\bar{k}$, then $\phi(\bar{k}, \bar{s})<0$, and hence we always have modulational stability in this case.

(iii) Possible existence of MI. MI may occur for nonzero wave numbers in higher-order gain bands. This property depends sensitively on the shape of the spectrum and the control parameter $\bar{s}$.

(iv) Number of gain bands. If the transversality condition $\partial_{\bar{k}} \phi \neq 0$ is satisfied at all the $N$ zeros of $\phi$ for a given $\bar{s}$ $>0$, then the number of gain bands $(m)$ is given by $N$ $=2 m(m=0,1, \ldots)$. This follows from Theorem $\mathrm{I}$ in the Appendix and the fact that $\phi(0, \bar{s})=1 / \bar{s}<0$ and $\phi \rightarrow$ $-\bar{k}^{2} / \bar{s}^{2}$ for $\bar{k} \rightarrow \infty$. Note that the stable case with no zeros, and hence no gain bands, is included $(m=0)$. 


\section{A. Higher-order gain bands}

Here we detail the properties of the higher-order MI gain bands that do not exist in the local limit $\sigma=0$, but may be formed through a bifurcation process when the system becomes sufficiently nonlocal. Let $\bar{s}_{b}<0$ denote the bifurcational value of the control parameter and assume that for $\bar{s}$ $<\bar{s}_{b}$, there are wave numbers $\bar{k}_{0}$ and $\bar{k}_{1}\left(\bar{k}_{0}<\bar{k}_{1}\right)$ satisfying the following properties $(i=0,1)$.

(a) $\phi\left(\bar{k}_{i}, \bar{s}\right)=0 \Leftrightarrow \hat{\Phi}\left(\bar{k}_{i}\right)=-\bar{k}_{i}^{2} /|\bar{s}|$.

(b) $\phi(\bar{k}, \bar{s})>0$ for $\bar{k}_{0}<\bar{k}<\bar{k}_{1} \Leftrightarrow \hat{\Phi}(\bar{k})<-\bar{k}^{2} /|\bar{s}|$ for $\bar{k}_{0}$ $<\bar{k}<\bar{k}_{1}$.

(c) $\partial_{\bar{k}} \phi\left(\bar{k}_{0}, \bar{s}\right)>0 \Leftrightarrow \hat{\Phi}^{\prime}\left(\bar{k}_{0}\right)<-2 \bar{k}_{0} /|\bar{s}|$.

(d) $\partial_{\bar{k}} \phi\left(\bar{k}_{1}, \bar{s}\right)<0 \Leftrightarrow \hat{\Phi}^{\prime}\left(\bar{k}_{1}\right)>-2 \bar{k}_{1} /|\bar{s}|$.

Again (c) and (d) are the transversality conditions at the ze$\operatorname{ros} \bar{k}_{0}$ and $\bar{k}_{1}$. All Eqs. (27)-(39) are formerly the same, except for sign changes in the equations that are in terms of real variables $k, \sigma, \rho_{0}$, and $g$. We have the following results:

(hd1) Gain profile. Equations (27)-(29) and the properties (f1) derived therefrom hold also for the defocusing case. However, the normalized gain is now

$$
g=\frac{k}{4 \rho_{0}} \sqrt{-4 \rho_{0} \hat{\Phi}(\bar{k})-k^{2}},
$$

and the velocity therefore becomes

$$
\frac{\partial g}{\partial \sigma}=-\left(\frac{k^{3}}{8 \rho_{0} g}\right) \hat{\Phi}^{\prime}(\bar{k})
$$

Thus the variation of the gain with the degree of nonlocality $\sigma$ still depends on the sign of $\hat{\Phi}^{\prime}(\bar{k})$, but now $\operatorname{sgn}\{d g / d \sigma\}$ $=-\operatorname{sgn}\left\{\hat{\Phi}^{\prime}(\bar{k})\right\}$.

(hd2) Bandwidth. The bandwidth $B$ is again defined as $B=k_{1}-k_{0}$ and Eq. (32) is still valid for both $k_{0}$ and $k_{1}$ due to the conditions (c) and (d). However, in real variables the velocities now become

$$
\begin{gathered}
\frac{\partial k_{i}}{\partial \sigma}=-\frac{2 \rho_{0} k_{i} \hat{\Phi}^{\prime}\left(\bar{k}_{i}\right)}{2 \rho_{0} \sigma \hat{\Phi}^{\prime}\left(\bar{k}_{i}\right)+k_{i}}, \\
\frac{\partial k_{i}}{\partial \rho_{0}}=\frac{k_{i}^{2} /\left(2 \rho_{0}\right)}{2 \rho_{0} \sigma \hat{\Phi}^{\prime}\left(\bar{k}_{i}\right)+k_{i}},
\end{gathered}
$$

which replaces Eqs. (33) and (34). From Eqs. (42) and (43) we see that both boundaries $k_{0}$ and $k_{1}$ have exactly the same dependences on $\sigma$ and $\rho_{0}$ as in the focusing case, given by the properties (hf2).

(hd3) Local minima and maxima. Equations (35)-(37) still apply, since they are in scaled variables. However, in real variables the velocity is now given by

$$
\frac{\partial k_{c}}{\partial \sigma}=-\frac{k_{c} \rho_{0} D\left(\bar{k}_{c}\right)}{\sigma \rho_{0} D\left(\bar{k}_{c}\right)+2 k_{c}},
$$

which replaces Eq. (38). Furthermore, in real variables, $k_{c}$ now corresponds to a local minimum when $\sigma \rho_{0} D\left(\bar{k}_{c}\right)<$ $-2 k_{c}$ and a local maximum when $\sigma \rho_{0} D\left(\bar{k}_{c}\right)>-2 k_{c}$. Nevertheless, a minimum still always decreases with $\sigma$ and the properties of a maximum still depends on the sign of $D\left(\bar{k}_{c}\right)$. If $-2 k_{c}<\sigma \rho_{0} D\left(\bar{k}_{c}\right)<0$, then $k_{c}$ will increase with $\sigma$, and if $\sigma \rho_{0} D\left(\bar{k}_{c}\right)>0$ then $k_{c}$ will decrease with $\sigma$. The properties of inflection points are the same as listed under (f3), except that now the inflection point occurs at $\sigma \rho_{0} D\left(\bar{k}_{c}\right)=-2 k_{c}$.

\section{EXPONENTIAL RESPONSE FUNCTION}

As a generic example of a response function with a positive-definite spectrum, we consider the typical exponential response function

$$
R(x)=\frac{1}{\sigma} \Phi\left(\frac{x}{\sigma}\right), \quad \Phi(\zeta)=\frac{1}{2} \exp (-|\zeta|),
$$

whose Fourier transform is a Lorentzian,

$$
\hat{R}(k)=\hat{\Phi}(\sigma k)=\frac{1}{1+\sigma^{2} k^{2}}
$$

This response function appears, e.g., in materials with a nonlinear response determined by a transport mechanism (diffusion, heat conduction, etc.) and described by the generic diffusionlike equation [20]

$$
\begin{aligned}
& i \partial_{z} \psi+\frac{1}{2} \partial_{x}^{2}+\Delta n \psi=0 \\
& \Delta n-\sigma^{2} \partial_{x}^{2} \Delta n=s|\psi|^{2}
\end{aligned}
$$

where $\sigma$ is the diffusion parameter. The Lorentzian spectrum (46) is a monotonically decreasing positive definite function. Thus MI is always present if the medium is focusing ( $s$ $=1$ ), whereas there can be no MI if the medium is defocusing $(s=-1)$, according to Ref. [26] and Table I. In the following we therefore consider only the focusing case $(s$ $=1, \bar{s}>0)$ :
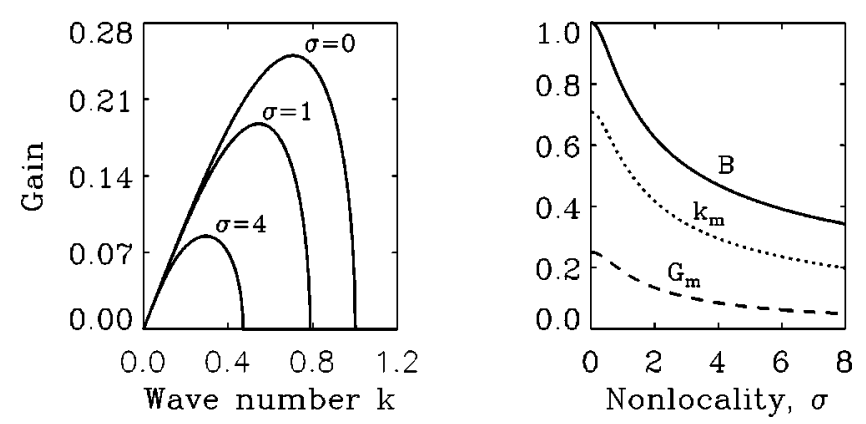

FIG. 1. Focusing nonlocal medium with $4 \rho_{0}=1$ and exponential response function. Left: MI gain versus wave number $k$ for $\sigma$ $=0,1,4$. Right: bandwidth $B$ (solid), maximum gain $G_{m}$ (dashed), and wave number $k_{m}$ at maximum gain (dotted) versus degree of nonlocality $(\sigma)$. 


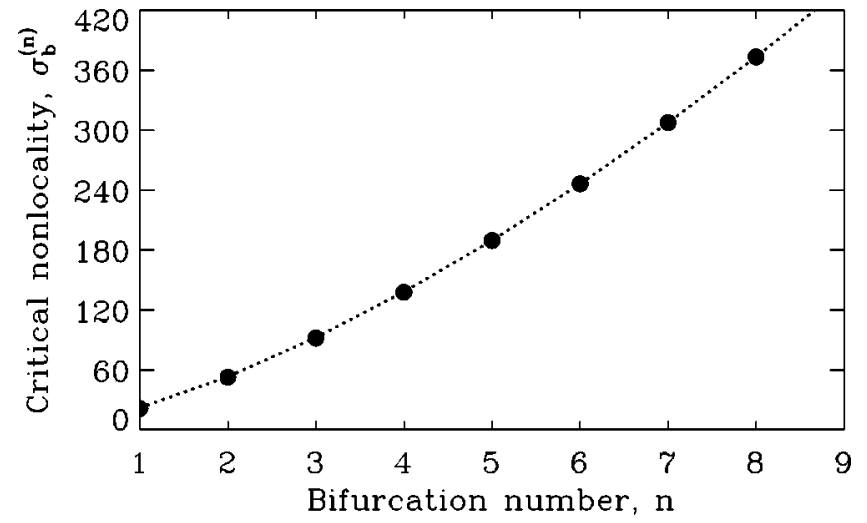

FIG. 2. Focusing nonlocal medium with $4 s \rho_{0}=1$ and rectangular response function. Bifurcation values of the nonlocality parameter $\sigma_{b}^{(n)}$ (dots) versus bifurcation number $n$. The dashed line shows approximation (52).

Let us summarize the generic properties predicted by our theory, given spectrum (46), for which $\hat{\Phi}(\bar{k})>0$ for all $\bar{k}$ and $\hat{\Phi}^{\prime}(\bar{k})<0$ for $\bar{k}>0$, with $\hat{\Phi}^{\prime}(0)=0$. First, we see that bifurcations cannot occur, since $\hat{\Phi}$ and $\hat{\Phi}^{\prime}$ have opposite sign, and thus the bifurcation Eqs. (23) and (24) do not have a solution, i.e., for all degrees of nonlocality $(\sigma)$, only the fundamental (long-wave) gain band exists. Equations (31) and (33) with $\hat{\Phi}^{\prime}(\bar{k})<0$ then predict that the maximum gain $G_{m}(\sigma)$ and the gain bandwidth $k_{1}=B(\sigma)$ always decrease with $\sigma$.

For more information on the gain profile, we must calculate the functions $D$ and $F$, i.e., we must calculate the specific curvature $\hat{\Phi}^{\prime \prime}(\bar{k})$ from Eq. (46). Inserting the curvature into Eqs. (28) and (29) gives that $D(\bar{k})<0$ and $F(\bar{k})<0$ for all $\bar{k}$, and thus the maximum $G_{m}$ of the fundamental gain band is always unique and occurs at the wave number $k_{c}=k_{m}$, which always decreases with $\sigma$ according to Eq. (38). From

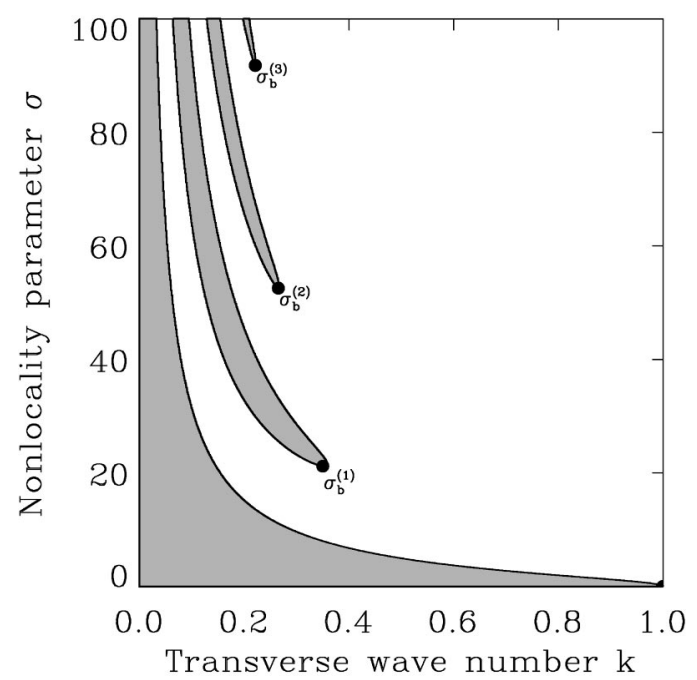

FIG. 3. Focusing nonlocal medium with $4 s \rho_{0}=1$ and rectangular response function. The first four $(n=0,1,2,3)$ MI gain bands (shaded areas) versus wave number $k$ and degree of nonlocality $(\sigma)$. Bifurcation points are indicated. the eigenvalue equation (12), we find the specific expressions for the bandwidth $B(\sigma)$ and maximum gain $G_{m}(\sigma)$ :

$$
B^{2}=\frac{\sqrt{1+16 \rho_{0} \sigma^{2}}-1}{2 \sigma^{2}}, G_{m}^{2}=\frac{\rho_{0} k_{m}^{2}}{1+\sigma^{2} k_{m}^{2}}-\frac{k_{m}^{4}}{4},
$$

where $k_{m}$ is the positive real solution of the equation $2 \sigma^{2} k_{m}^{3}+2 k_{m}=\sqrt{8 \rho_{0}}$.

We have numerically investigated the eigenvalue equation (12) and depicted the results in Fig. 1. The numerical results and the expressions for $B(\sigma), G_{m}(\sigma)$, and $k_{m}(\sigma)$ confirm the generic properties listed in Tables III and I, i.e., only one fundamental MI gain band exists with a bandwidth, unique maximum, and wave number at maximum gain that all decrease with the degree of nonlocality. Thus the nonlocality tends to suppress MI, but can never remove it entirely. Using the qualitatively similar Gaussian response function, these results were confirmed by direct numerical simulation in Ref. [26]. There the exponential response was also considered, but without finding the analytical expressions for the maximum gain and bandwidth, and without any theory for the generic properties of the gain spectrum.

\section{RECTANGULAR RESPONSE FUNCTION}

Now, let us consider the situation where the spectrum is not sign definite and not strictly decreasing for all $k$. Then it is possible to have additional gain bands in both the focusing and defocusing cases. As an example, we consider the rectangular response function

$$
R(x)=\frac{1}{\sigma} \Phi\left(\frac{x}{\sigma}\right), \quad \Phi(\zeta)= \begin{cases}\frac{1}{2}, & |\zeta| \leqslant 1 \\ 0, & |\zeta|>1,\end{cases}
$$

whose Fourier transform is given by

$$
\hat{R}(k)=\hat{\Phi}(\sigma k)=\frac{\sin (k \sigma)}{k \sigma} .
$$

The bifurcation equation (23) then reads

$$
3 \tan \left(\bar{k}_{b}\right)=\bar{k}_{b}, \quad \bar{k}_{b}>0, \quad s \sin \left(\bar{k}_{b}\right)>0,
$$

from which one finds the bifurcation values $\sigma_{b}$ and $k_{b}$ of the degree of nonlocality and the wave number,

$$
\sigma_{b}^{2}=\frac{\bar{k}_{b}^{3}}{4 s \rho_{0} \sin \left(\bar{k}_{b}\right)}, \quad k_{b}=\frac{\bar{k}_{b}}{\sigma_{b}} .
$$

The bifurcation relation (49) gives the approximate value $\bar{k}_{b}^{(n)}$ for the $n$th bifurcation,

$$
\bar{k}_{b}^{(n)} \simeq\left(2 n+\frac{s}{2}\right) \pi, \quad n=1,2,3, \ldots,
$$

corresponding to the degree of nonlocality and the transverse wave number 
TABLE IV. Nonlocal focusing medium with a rectangular response function. Lowest-order bifurcations for $4 s \rho_{0}=1$.

\begin{tabular}{lccc}
\hline \hline Nonlocality & Eq. (52) & Wave number & Eq. (52) \\
\hline$\sigma_{b}^{(1)}=21.203$ & $\sigma_{b}^{(1)} \simeq 22.011$ & $k_{b}^{(1)}=0.352$ & $k_{b}^{(1)} \simeq 0.357$ \\
$\sigma_{b}^{(2)}=52.555$ & $\sigma_{b}^{(2)} \simeq 53.155$ & $k_{b}^{(2)}=0.263$ & $k_{b}^{(2)} \simeq 0.266$ \\
\hline \hline
\end{tabular}

$$
\sigma_{b}^{(n)} \simeq \sqrt{\frac{\left(2 n+\frac{s}{2}\right)^{3} \pi^{3}}{4 \rho_{0}}}, \quad k_{b}^{(n)} \simeq \sqrt{\frac{4 \rho_{0}}{\left(2 n+\frac{s}{2}\right) \pi}}
$$

Strictly speaking, Eq (49) has also the zero solution $\bar{k}_{b}=0$, corresponding to $\sigma_{b}=0$, but we do not count this as a bifurcation. Moreover, one finds that the curvature of the structural function $\phi$ is always negative at the bifurcation points,

$$
\phi^{\prime \prime}\left(\bar{k}_{b}\right)=-\left(\frac{6+\bar{k}_{b}^{2}}{\bar{s}_{b}^{2}}\right)<0 .
$$

Thus new MI bands are always excited at the bifurcation, both when the nonlinearity is focusing and defocusing.

In a gain band, $\phi>0$; and thus $\bar{s} \sin (\bar{k})>0$. From the definition $\partial_{\bar{k}} g\left(\bar{k}_{c}, \bar{s}\right)=0$ of the critical wave number $\bar{k}_{c}$, we further have that $\bar{s}\left[\sin \left(\bar{k}_{c}\right)+\bar{k}_{c} \cos \left(\bar{k}_{c}\right)\right]=4 \bar{k}_{c}^{2}>0$. Using these relations in the definition (29) of the function $D$, we find that $\bar{s} D\left(\bar{k}_{c}\right)<0$. Thus $F$ is always negative on the critical points (or extrema of the gain profile) and all gain bands therefore have a unique maximum. From Eqs. (38) and (44) we then have that the wave number $k_{c}=k_{m}^{(n)}$ at maximum gain of the $n$th gain band will always decrease with the degree of nonlocality $(\sigma)$, both in focusing and defocusing media. Let us look at these cases separately in more detail.

\section{A. Focusing case $(s=+1)$}

In the focusing case the fundamental gain band $(n=0)$ is always present, and thus MI is always present as well, in accordance with Table I. For the response function (48), the
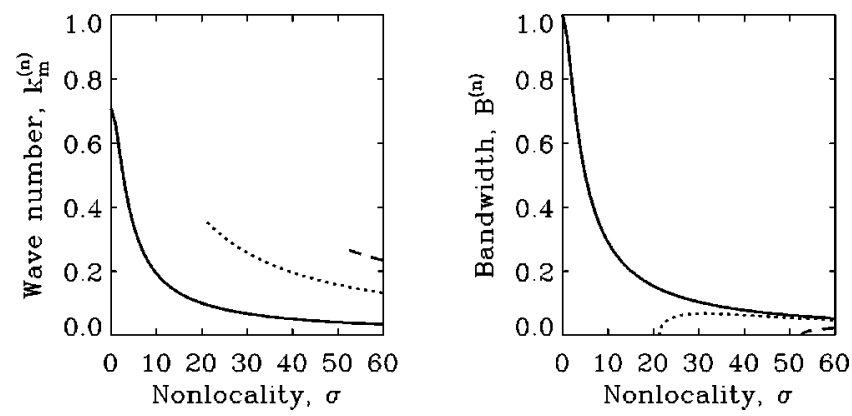

FIG. 4. Focusing nonlocal medium with $4 s \rho_{0}=1$ and rectangular response function. Wave number at maximum gain $k_{m}^{(n)}$ (left) and bandwidth $B^{(n)}$ (right) of bands $n=0$ (solid), $n=1$ (dotted), and $n=2$ (dashed).

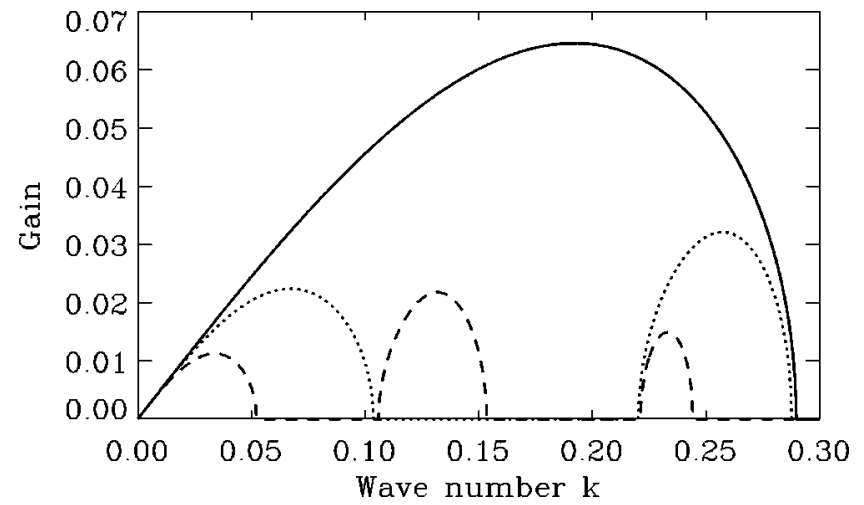

FIG. 5. Focusing nonlocal medium with $4 s \rho_{0}=1$ and rectangular response function. Gain versus modulation wave number $k$ for $\sigma=10$ (solid), $\sigma=30$ (dotted), and $\sigma=60$ (dashed).

bandwidth $\bar{k}$ of the fundamental gain band is always less than $\pi$, and thus both $\hat{\Phi}^{\prime}, D$, and $F$ are negative for all 0 $<\bar{k}<\bar{k}_{1}$. The fundamental gain band therefore has a unique maximum gain; and according to Eqs. (31), (33), and (38) its maximum gain, its bandwidth, and its wave number at maximum gain all decrease with the degree of nonlocality $(\sigma)$. For the higher-order gain bands the generic properties of the bandwidth $B^{(n)}$ and the maximum gain $G_{m}^{(n)}$ are difficult to predict.

The results for the first and second bifurcation are summarized in Table IV, whereas all bifurcation values of the nonlocality are plotted in Fig. 2. We see that Eq. (52) gives a quite accurate prediction of the bifurcations. The accuracy becomes better with increasing $n$.

The full structure of the gain spectrum is depicted as a contour plot in Fig. 3. For small and moderate values of the degree of nonlocality $(\sigma<21.2)$, we only have one fundamental MI gain band. For higher values of $\sigma$ new gain bands appear as predicted. These results are consistent with the findings in Ref. [26].

The dependence of $k_{m}^{(n)}$ and $B^{(n)}$ on $\sigma$ are depicted in Fig. 4 , which confirms the predicted generic properties and further shows that the bandwidth of the different bands approach each other when $\sigma$ increases.

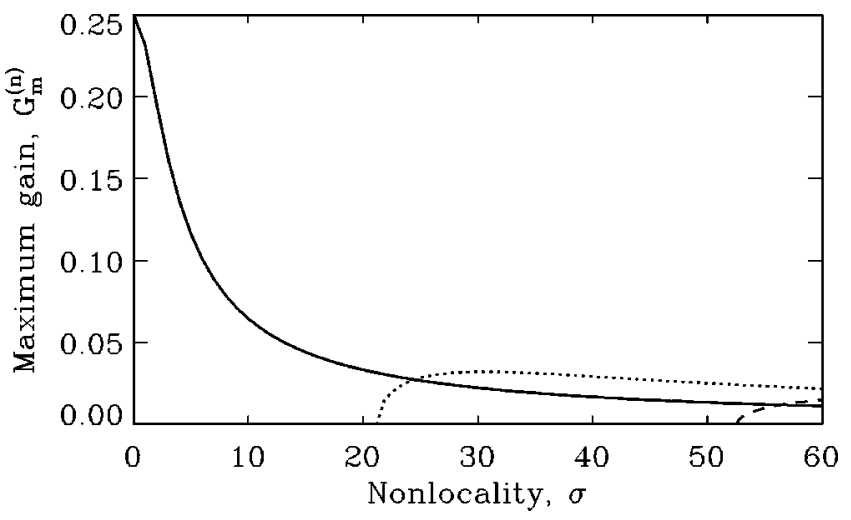

FIG. 6. Focusing nonlocal medium with $4 s \rho_{0}=1$ and rectangular response function. Maximum gain $G_{m}^{(n)}$ versus $\sigma$ for bands $n$ $=0$ (solid), $n=1$ (dotted), and $n=2$ (dashed). 
TABLE V. Nonlocal defocusing material with a rectangular response function. Lowest-order bifurcations for $4 s \rho_{0}=-1$.

\begin{tabular}{llll}
\hline \hline Nonlocality & Eq. (52) & Wave number & Eq. (52) \\
\hline$\sigma_{b}^{(1)}=9.176$ & $\sigma_{b}^{(1)} \simeq 10.230$ & $k_{b}^{(1)}=0.444$ & $k_{b}^{(1)} \simeq 0.461$ \\
$\sigma_{b}^{(2)}=35.780$ & $\sigma_{b}^{(2)} \simeq 36.461$ & $k_{b}^{(2)}=0.300$ & $k_{b}^{(2)} \simeq 0.302$ \\
\hline \hline
\end{tabular}

In Fig. 5 we have shown cuts of the MI gain spectrum at $\sigma=10,30$, and 60 . We note a surprising feature for $\sigma=30$, which is not predicted by our analysis of the generic features: the maximum gain of the second band is higher than that of the fundamental band. To show this in more detail, we have plotted the maximum gain in Fig. 6 . Here we clearly see that at $\sigma=25(\sigma=56)$ the maximum gain of the $n=1 \quad(n=2)$ band becomes larger than the maximum gain of the fundamental $n=0$ band. This crossing should be detectable in a numerical experiment, since the system will always eventually select the wave number at maximum gain when starting from random white noise.

\section{B. Defocusing case $(s=-1)$}

In the defocusing case, there is no fundamental $(n=0)$ gain band, and thus all plane waves are modulationally stable until the nonlocality becomes sufficiently strong to reach the first bifurcation. Table $\mathrm{V}$ summarizes the results for the first and second bifurcation, whereas all bifurcation values of the nonlocality are plotted in Fig. 7. We see that Eq. (52) gives a quite accurate prediction of the bifurcations also in the defocusing case. The accuracy again becomes better for increasing $n$.

The full structure of the gain spectrum is depicted as a contour plot in Fig. 8. The fundamental $n=0$ MI gain band is absent, so for small and moderate values of the degree of nonlocality, $\sigma<9.2$, there is no MI. For higher values of $\sigma$ $>9.2$ gain bands appear as predicted. These results are consistent with the preliminary findings in Ref. [26].

The dependence of $k_{m}^{(n)}$ and $B^{(n)}$ on $\sigma$ are depicted in Fig. 9 , which again confirms the predicted generic properties and

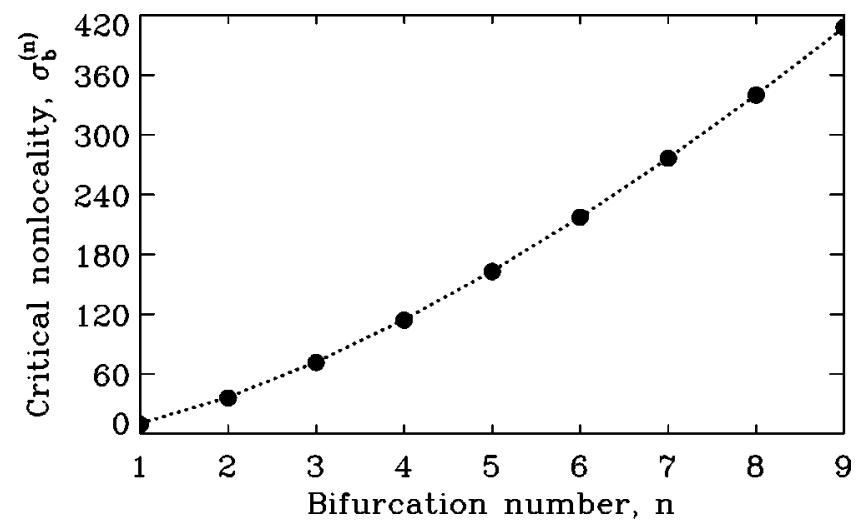

FIG. 7. Defocusing nonlocal medium with $4 s \rho_{0}=-1$ and rectangular response function. Bifurcation values of the nonlocality parameter $\sigma_{b}^{(n)}$ (dots) versus bifurcation number $n$. The dashed line shows approximation (52).

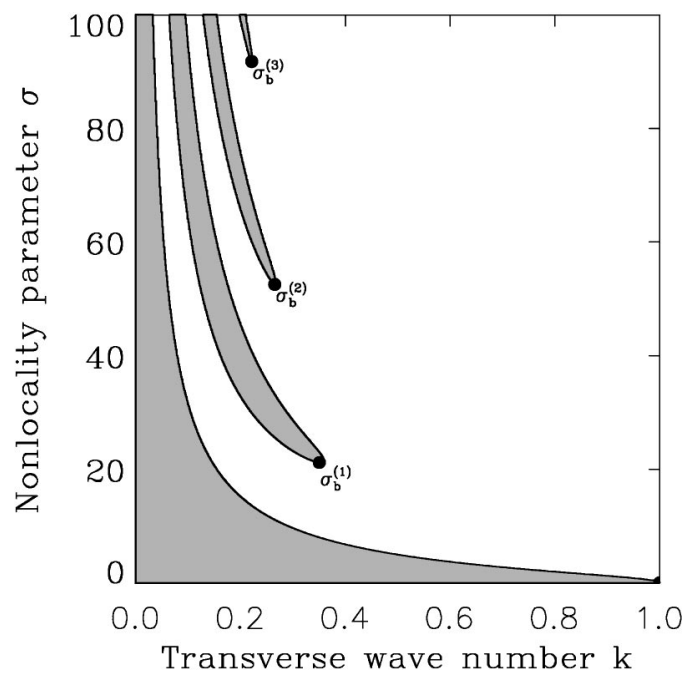

FIG. 8. Defocusing nonlocal medium with $4 s \rho_{0}=-1$ and rectangular response function. The first three $(n=1,2,3)$ MI gain bands (shaded areas) versus wave number $k$ and degree of nonlocality $\sigma$. Bifurcation points are indicated.

shows that the bandwidths of the different bands approach each other when $\sigma$ increases.

In Fig. 10 we have shown cuts of the gain spectrum at $\sigma=20$, 50, and 80. Again, for both $\sigma=50$ and 80, we observe that the maximum gain of the $n=2$ band is higher than that of the lowest $n=1$ band. To show this in more detail, we have plotted the maximum gain in Fig. 11.

From Fig. 11 we clearly see that at $\sigma \approx 47(\sigma \approx 84)$ the maximum gain of the $n=2(n=3)$ band becomes larger than the maximum gain of the lowest $n=1$ band. We even see that at $\sigma \approx 109$, the third $n=3$ band becomes dominant, i.e., its maximum gain exceeds also that of the second $n=2$ band. Again these crossings should be detectable in a numerical experiment, since the system will always eventually select the wave number at maximum gain when starting from random white noise.

In fact, our numerical simulations clearly confirm this prediction. We numerically integrated nonlocal NLS equation (1) with the defocusing nonlinearity and the rectangular response function in the form of Eq. (47). As the initial conditions, we used a planar wave front superimposed with a
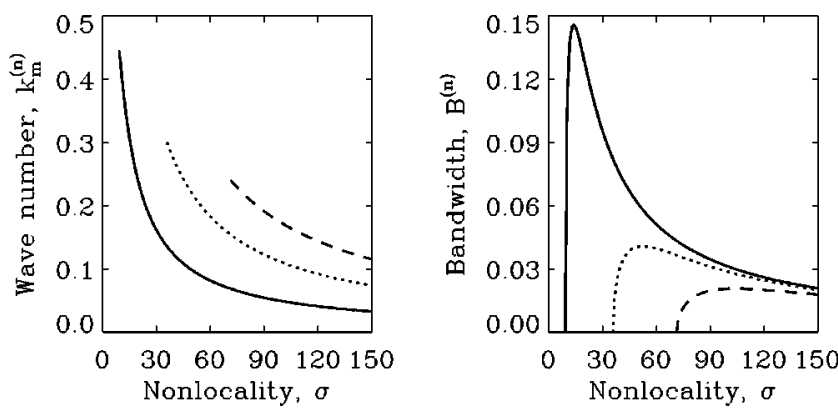

FIG. 9. Defocusing nonlocal medium with $4 s \rho_{0}=-1$ and rectangular response function. Wave number at maximum gain $k_{m}^{(n)}$ (left) and bandwidth $B^{(n)}$ (right) of bands $n=1$ (solid), $n=2$ (dotted), and $n=3$ (dashed). 


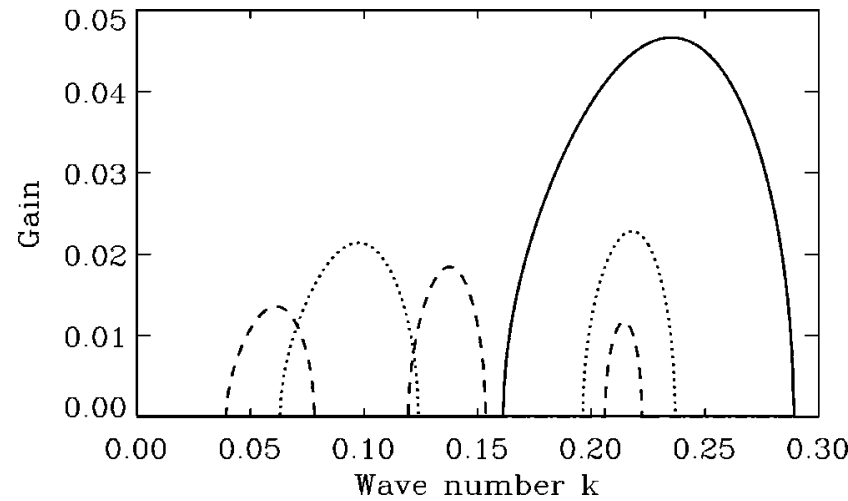

FIG. 10. Defocusing nonlocal medium with $4 s \rho_{0}=-1$ and rectangular response function. Gain versus wave number $k$ for $\sigma$ $=20$ (solid), $\sigma=50$ (dotted), and $\sigma=80$ (dashed).

weak (less than $10^{-5}$ ) random perturbation. Then the equation was numerically integrated using the split-step fast Fourier method with a transverse resolution of $\Delta x=10^{-2}$ and a step length of $\Delta z=10^{-3}$.

Figure 12 summarizes the results of this numerical experiment for $s \rho_{0}=-1$. It shows the wave number at maximum gain in propagation as a function of the nonlocality parameter $\sigma$. Lines correspond to solutions obtained from dispersion relation (12). Squares indicate the results of full numerical simulations of Eq. (1). The agreement between the small signal analysis and numerical simulation is excellent. The inset shows examples of the development of the instability for three values of the nonlocality parameter $\sigma$. It is evident

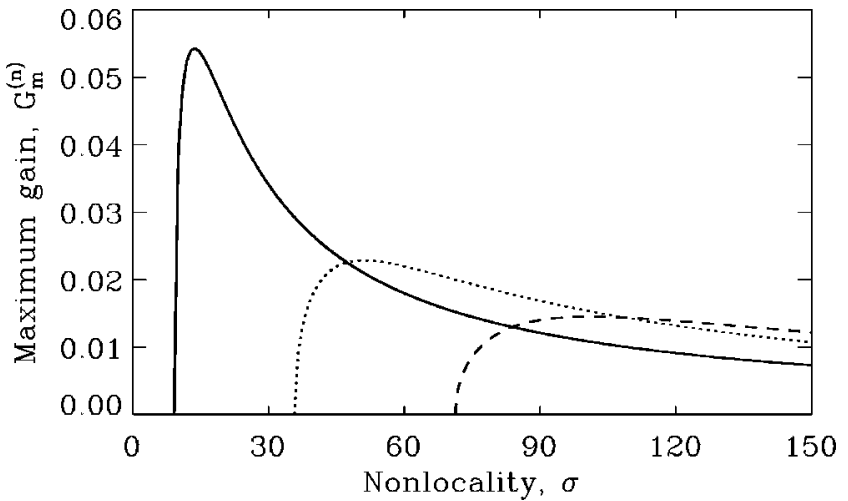

FIG. 11. Defocusing nonlocal medium with $4 s \rho_{0}=-1$ and rectangular response function. Maximum gain $G_{m}^{(n)}$ versus degree of nonlocality for bands $n=1$ (solid), $n=2$ (dotted), and $n=3$ (dashed).

that as the degree of nonlocality increases, the period of the MI signal first increases until the growth rate of the fundamental and second MI bands equalize ( $\sigma \approx 24$ in Fig. 12). Subsequent increase of $\sigma$ leads to instability with shorter period, which indicates that the system follows the path determined by the second MI band.

\section{CONCLUSION}

The linear stage of the MI for the nonlocal NLS equation has been studied in terms of the spectrum of the response

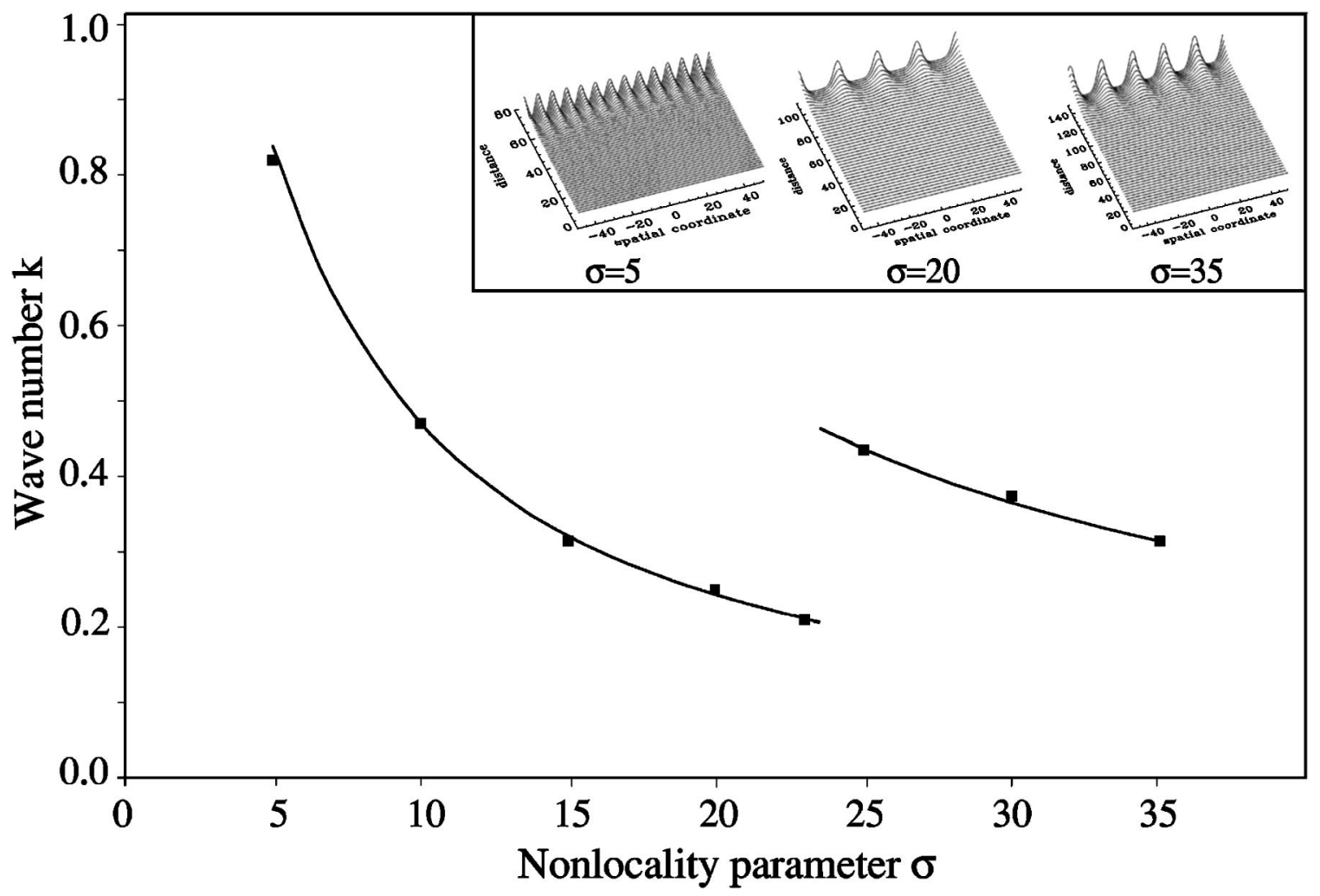

FIG. 12. Wave number at maximum gain versus the degree of nonlocality $(\sigma)$. Defocusing nonlocal medium with $s \rho_{0}=-1$ and rectangular response function. Lines, solutions to dispersion relation (12); squares, results of direct numerical integration. 
function. From dispersion relation (12), it follows that the crucial point in this discussion is the location of the spectrum of the response function relative to the parabola $k^{2}$ in $k$ space. The following features complement and extend the results obtained in Ref. [26]:

(i) The MI is of the finite bandwidth type. It consists of a finite number of well-separated gain bands. Moreover, it is possible to predict the occurrence of excitation, vanishing, coalescence, and separation of MI bands.

(ii) For a large class of response functions (exponential, square, Gaussian, etc.), each MI band has a unique maximum growth rate. In isolation, each band resembles the MI band found in the focusing local NLS equation.

(iii) In the focusing case we always find at least one MI gain band centered at about $k=0$. It is verified analytically that the width of this MI band, as well as the corresponding growth rate, decreases when increasing the width of the response function, provided the spectrum of the response function is decreasing in this MI band. Furthermore, additional MI bands are excited at higher wave numbers when the width parameter exceeds a certain threshold, i.e., when the nonlinearity becomes sufficiently nonlocal. The latter phenomenon is a unique feature of the nonlocal nonlinearity and has no equivalent in the local case and the weakly nonlocal limit.

(iv) In the defocusing case we can either have stability or MI of the finite bandwidth type. The latter situation can only occur in the high wave number regime, and only if the width of the response function exceeds a certain threshold, i.e., when the nonlinearity becomes sufficiently nonlocal.

(v) In both the focusing and defocusing case, the higherorder MI bands move towards lower wave numbers as the width of the response function increases. In the limit of strong nonlocality, the MI bands vanish completely. This result agrees with the fact that the strongly nonlocal limit of the NLS model (1) is a linear model.

(vi) Finally, it should be emphasized that the results of small signal analysis are in complete agreement with those obtained by direct numerical integration of the nonlocal NLS equation describing the general type of nonlocal nonlinear media that we have studied.

\section{ACKNOWLEDGMENTS}

This work was supported by the Danish Technical Research Council (STVF Grant No. 26-00-0355), the Danish Natural Sciences Foundation (SNF Grant No. 9903273), and the Graduate School in Nonlinear Science (The Danish Research Academy).

\section{APPENDIX: SUFFICIENT CONDITION FOR THE EXISTENCE OF A FINITE NUMBER OF GAIN BANDS}

Let us assume that the function $\phi$ defined by Eq. (16), i.e.,

$$
\phi(\bar{k}, \bar{s}) \equiv \frac{\bar{s} \hat{\Phi}(\bar{k})-\bar{k}^{2}}{\bar{s}^{2}}
$$

changes sign. Then we have the following important result:

Theorem 1. Let $\bar{s}$ be a given finite, real number. If the transversality condition $\partial_{\bar{k}} \phi \neq 0$ is satisfied for all the zeros of $\phi$, then these points are distinct and isolated. Moreover, the number of such zeros is finite.

Proof. First, let us prove that the zeros of $\phi$ are distinct and isolated. We proceed by means of a contradiction argument. Let $\left\{\bar{k}_{n}\right\}_{n=1}^{\infty}$ be a convergent sequence of zeros of $\phi$, i.e., $\phi\left(\bar{k}_{n}, \bar{s}\right)=0$ for all $n$ and $\lim _{n \rightarrow \infty} \bar{k}_{n}=\bar{k}_{*}$. Then, by continuity of $\phi$, we have

$$
0=\lim _{n \rightarrow \infty} \phi\left(\bar{k}_{n}, \bar{s}\right)=\phi\left(\bar{k}_{*}, \bar{s}\right)
$$

which means that the accumulation point $\bar{k}_{*}$ is a zero of $\phi$. Hence any interval about $\bar{k}_{*}$ contains at least one zero $\bar{k}_{n}$. But the transversality condition implies that $\partial_{\bar{k}} \phi\left(\bar{k}_{*}, \bar{s}\right)$ $\neq 0$, from which it follows that there is an open interval $I$ $=\left\langle\bar{k}_{*}-\Delta \bar{k}, \bar{k}_{*}+\Delta \bar{k}\right\rangle$, such that $\phi(\bar{k} ; \bar{s}) \neq 0$ for all $\bar{k} \in I, \bar{k}$ $\neq \bar{k}_{*}$, from which it follows that no zero of $\phi$ can be an accumulation point of some sequence of zeros of $\phi$. Therefore, all the zeros must be distinct and isolated. Next, let us prove that the set of zeros is finite. From the RiemannLebesques lemma we have that $\lim _{n \rightarrow \infty} \hat{\Phi}(\bar{k})=0$, and hence due to the definition of $\phi$ we have that $\phi(\bar{k} ; \bar{s}) \sim-\left(\bar{k}^{2} / \bar{s}^{2}\right)$ as $\bar{k} \rightarrow \infty$. Therefore there must be a $\bar{k}_{B}$ such that $\phi\left(\bar{k}_{B}, \bar{s}\right)=0$ and $\phi(\bar{k}, \bar{s})<0$ for all $\bar{k}>\bar{k}_{B}$. Hence all the zeros of $\phi$ belong to the bounded, half-open interval $\left\langle 0, \bar{k}_{B}\right]$. Hence we can only have a finite sequence $\left\{\bar{k}_{n}\right\}_{n=1}^{N}, \bar{k}_{N} \equiv \bar{k}_{B}$ for which $\phi\left(\bar{k}_{n}, \bar{s}\right)=0, n=1,2, \ldots, N$.

We immediately obtain the following result: Let $\bar{s}$ be a given finite real number and assume that the transversality condition $\partial_{\bar{k}} \phi \neq 0$ for all the zeros of $\bar{k}_{n}(n=1,2, \ldots, N)$ of $\phi$. Then the number of zeros $(N)$ is odd (even) for the case $\bar{s}>0 \quad(\bar{s}<0)$. This follows from the fact that we have $\phi(0 ; \bar{s})>0[\phi(0 ; \bar{s})<0]$ for $\bar{s}>0 \quad(\bar{s}<0)$ and $\phi(\bar{k} ; \bar{s}) \sim$ $-\left(\bar{k}^{2} / \bar{s}^{2}\right)$ as $\bar{k} \rightarrow \infty$. In the situation described by Theorem 1 we have also a counting rule for the number of gain bands as a function of the number of transversal cuttings. In the focusing case $(\bar{s}>0)$, we have $N=2 m-1 \quad(m=1,2, \ldots)$, while in the defocusing case $N=2 m(m=0,1,2, \ldots)$. In both cases the number $m$ is equal to the number of finite, well-separated gain bands. Notice that we allow for the case $m=0$ in the defocusing case, which accounts for the case where there are no gain bands, i.e., the modulational stable situation. 
[1] T.B. Benjamin and J.E. Feir, J. Fluid Mech. 27, 417 (1967).

[2] A. Hasegawa, Plasma Instabilities and Nonlinear Effects (Springer-Verlag, Heidelberg, 1975).

[3] L.A. Ostrovskii, Zh. Eksp. Teor. Fiz. 51, 1189 (1966) [Sov. Phys. JETP 24, 797 (1967)].

[4] V.I. Bespalov and V.I. Talanov, Pis'ma Zh. Eksp. Teor. Fiz. 3, 471 (1966) [JETP Lett. 3, 307 (1966)]; V.I. Karpman, ibid. 6, 829 (1967) [6, 277 (1967)].

[5] Yu.S. Kivshar and M. Peyrard, Phys. Rev. A 46, 3198 (1992).

[6] P.D. Miller and O. Bang, Phys. Rev. E 57, 6038 (1998).

[7] S. Trillo, S. Wabnitz, G.I. Stegeman, and E.M. Wright, J. Opt. Soc. Am. B 6, 889 (1989).

[8] M.J. Potasek, Opt. Lett. 12, 921 (1987)

[9] Yu.S. Kivshar, D. Anderson, and M. Lisak, Phys. Scr. 48, 679 (1993).

[10] M. Soljacic, M. Segev, T. Coskun, D. Christodoulides, and A. Vishwanath, Phys. Rev. Lett. 84, 467 (2000).

[11] J. Wyller, Physica D 157, 90 (2001).

[12] J.P. Gordon, R.C. Leite, R.S. Moore, S.P. Porto, and J.R. Whinnery, J. Appl. Phys. 36, 3 (1965).

[13] S. Akhmanov, D.P. Krindach, A.V. Migulin, A.P. Sukhorukov, and R.V. Khokhlov, IEEE J. Quantum Electron. QE-4, 568 (1968).

[14] M. Horovitz, R. Daisy, O. Werner, and B. Fischer, Opt. Lett. 17, 475 (1992).

[15] I.A. Kolchugina, V.A. Mironov, and A.M. Sergeev, Pis'ma Zh. Eksp. Teor. Fiz. 31, 333 (1980) [JETP Lett. 31, 304 (1980)]; V.A. Mironov, A.M. Sergeev, and E.M. Sher, Dokl Akad. Nauk. USSR 260, 325 (1981) [Sov. Phys. Dokl. 26, 861 (1981)].

[16] D. Suter and T. Blasberg, Phys. Rev. A 48, 4583 (1993).

[17] M.V. Porkolab and M.V. Goldman, Phys. Fluids 19, 872 (1976).
[18] A.G. Litvak and A.M. Sergeev, Pis'ma Zh. Eksp. Teor. Fiz 27, 549 (1978) [JETP Lett. 27, 517 (1978)].

[19] T.A. Davydova and A.I. Fishchuk, Ukr. J. Phys. 40, 487 (1995).

[20] A.G. Litvak, V.A. Mironov, G.M. Fraiman, and A.D. Yunakovskii, Fiz. Plazmy 1, 60 (1975) [Sov. J. Plasma Phys. 1, 31 (1975)].

[21] H.L. Pecseli and J.J. Rasmussen, Plasma Phys. 22, 421 (1980).

[22] F. Dalfovo, S. Giorgini, L.P. Pitaevskii, and S. Stringari, Rev. Mod. Phys. 71, 463 (1999); K. Goral, K. Rzazewski, and T. Pfau, Phys. Rev. A 61, 051601(R) (2000); V.M. Perez-Garcia, V. V. Konotop, and J.J. Garcia-Ripoll, Phys. Rev. E 62, 4300 (2000).

[23] D.W. McLaughlin, D.J. Muraki, and M.J. Shelley, Physica D 97, 471 (1996).

[24] M. Peccianti, K.A. Brzdakiewicz, and G. Assanto, Opt. Lett. 27, 1460 (2002); M. Peccianti, A. De Rossi, G. Assanto, A. De Luca, C. Umeton, and I.C. Khoo, Appl. Phys. Lett. 77, 7 (2000).

[25] O. Bang, W. Krolikowski, J. Wyller, and J.J. Rasmussen, Phys. Rev. E 66, 046619 (2002).

[26] W. Krolikowski, O. Bang, J.J. Rasmussen, and J. Wyller, Phys. Rev. E 64, 016612 (2001).

[27] A. Parola, L. Salanich, and L. Reatto, Phys. Rev. A 57, R3180 (1998).

[28] X. Wang, D.W. Brown, K. Lindenberg, and B.J. West, Phys. Rev. A 37, 3557 (1988).

[29] A. Nakamura, J. Phys. Soc. Jpn. 42, 1824 (1977).

[30] W. Krolikowski and O. Bang, Phys. Rev. E 63, 016610 (2001).

[31] A. Snyder and J. Mitchell, Science 276, 1538 (1997).

[32] G.B. Folland, Real Analysis. Modern Techniques and Their Applications (Wiley, New York, 1984), p. 241. 